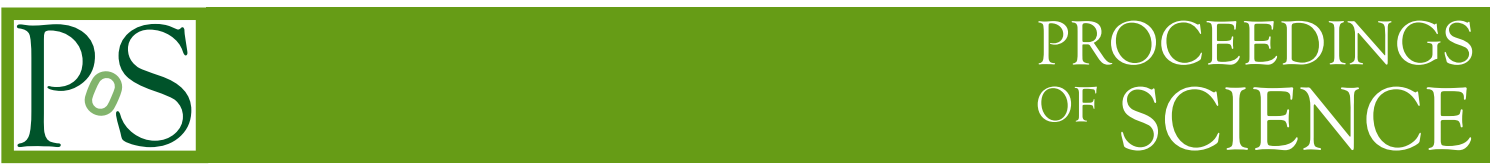

\title{
The $\Upsilon \rightarrow X \gamma$ photon spectrum in the soft-energy region
}

\section{Pedro Ruiz-Femenía*}

Institut für Theoretische Physik E, RWTH Aachen University, D-52056 Aachen, Germany

E-mail: ruiz@physik.rwth-aachen.de

\begin{abstract}
The dominant contribution of the next-to-leading order perturbative QCD corrections to the $\Upsilon \rightarrow$ $X \gamma$ photon energy spectrum for photon energies $\omega \sim m_{b} \alpha_{s}$ can be obtained trivially from the knowledge of the corresponding $\mathscr{O}(\alpha)$ correction to the positronium decay spectrum in the same region. The latter can be obtained from the NRQED computation of the orthopositronium photon spectrum, or by applying the threshold expansion to the 1-loop QED amplitude, as it is explained in this talk.
\end{abstract}

International Workshop on Effective Field Theories: from the pion to the upsilon February 2-6 2009

Valencia, Spain

*I wish to thank the organizers of EFT09 for the excellent arrangements and warm hospitality. This work is supported in part by the DFG Sonderforschungsbereich/Transregio 9 "Computergestützte Theoretische Teilchenphysik", by the European Commission MRTN FLAVIAnet under Contract No. MRTN-CT-2006-035482, and by the Ministerio de Ciencia e Innovación under Grant No. FPA2007-60323. 
Apart from being of physical relevance in itself, the Positronium system provides a testing ground for the effective field theory (EFT) concepts and techniques devised in the study of radiative decay amplitudes, that could eventually be applied to the description of the more intricate radiative decays of heavy quarkonia $(Q \bar{Q} \rightarrow X \gamma)$. An example of the latter is provided in this talk, where we analyze the region of photon energies $\omega \sim m \alpha$ (soft-energy region) in the radiative decay of a heavy fermion-antifermion system through the study of the $3 \gamma$ decay of the Positronium spin triplet ground state (orthopositronium: o-Ps). In particular we show that in this regime the next-to-leading order (NLO) corrections to the direct decay photon spectrum of a color singlet $Q \bar{Q}$ pair in angularmomentum configuration ${ }^{3} S_{1}$ are just given by the corresponding correction to the o-Ps photon spectrum up to a trivial color factor. The soft-energy region is accessible in the o-Ps $\rightarrow 3 \gamma$ decay because one of the final state photons can have an arbitrarily small energy, the other two being hard photons with energy $\lesssim m$. The process can be then viewed as the radiative version of the o-Ps $\rightarrow X$ decay, where $X=2 \gamma$.

The computation of the $\mathscr{O}(\alpha)$ corrections to the o-Ps spectrum in the soft-energy region can be considerably simplified using EFT methods or through the asymptotic expansion of the relevant 1-loop diagrams. While the latter technique generalizes straightforwardly to the corresponding computation for quarkonium radiative decays, the EFT framework naturally explains the origin of the dominant term as a consequence of the non-relativistic nature of the system.

\section{NRQED computation of the o-Ps decay spectrum for $\omega \ll m$}

The Non-Relativistic QED (NRQED) framework used in Refs. [1,2] provides a systematic way to compute bound state effects in the the o-Ps decay spectrum when the photon energy is much smaller than the electron mass. Contrary to the usual relativistic approach where the 3photon annihilation is considered to take place at very short distances as compared to the range of the electromagnetic binding force between $e^{+} e^{-}$, the non-relativistic description takes into account that there is a long-distance part in the o-Ps decay process when one the photons in the final state is not hard $(\omega \ll m)$. In the latter case, the decay proceeds in two steps: the low-energy photon is first radiated from the bound state, making a transition from the C-odd ground state o-Ps $\left({ }^{3} S_{1}\right)$ to a C-even Positronium state $n$, which subsequently decays into two photons (see the EFT graph of Fig. 1). The emission of the low energy photon from o-Ps is described by the Coulomb Hamiltonian of the $e^{+} e^{-}$system in interaction with a quantized electromagnetic field [1,2].

Since the computation of the photon spectrum in Ref. [1] was intended for photon energies comparable to the Positronium binding energy, $\omega \sim m \alpha^{2}$, the interaction Hamiltonian was used in the dipole approximation limit. This approximation amounts to evaluating the photon vector potential $\mathbf{A}$ in the center of mass of the Positronium system, which is fully legitimate for radiated photons with wavelengths much larger than the characteristic size of the Positronium atom $(a=2 / m \alpha)$. Higher order multipoles arise as a Taylor series in the relative coordinate $\mathbf{x}$ and are suppressed by powers of $\omega / a$ under the assumption that the relevant amplitudes one has to compute involve integrations to spatial extents of order $\sim a$. For photons with larger energies, $\omega \gg m \alpha^{2}$, this premise will invalidate the use of the multipole expansion.

However, in the case of the o-Ps system that undergoes a radiative transition before decaying, and as it was properly pointed out by Voloshin [3], the scale $1 / a$ does not constraint the maximum 


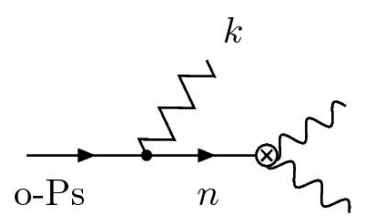

Figure 1: NRQED graph for the o-Ps $\rightarrow 3 \gamma$ decay. The zig-zag line represents the low-energy photon.

photon energy for which we can apply the multipole expansion. The reason is that after the soft photon is radiated we have to consider all possible $e^{+} e^{-}$intermediate states with the right quantum numbers. The propagation of these $e^{+} e^{-}$states is described by the Green function obtained from the Coulomb Hamiltonian,

$$
\left(H_{C}+\frac{\kappa^{2}}{m}\right) G(\mathbf{x}, \mathbf{y}, \kappa)=\delta(\mathbf{x}-\mathbf{y})
$$

at energy $\kappa^{2} / m \simeq \omega-E_{0}$. Since the annihilation of the intermediate $e^{+} e^{-}$pair into two photons takes place at small distances, the amplitude for the full process is thus given by a convolution of the o-Ps ground state and the Green function. In coordinate space, the Green function has a characteristic size ruled by the exponential factor $\exp (-\kappa r)$. We note that for soft photons of energy $m \alpha^{2} \ll \omega \ll m$, this exponential factor constrains the relevant integration region to distances of order $\kappa^{-1} \sim 1 / \sqrt{m \omega}$, much smaller than the spatial extent of the initial Positronium atom. Therefore, the characteristic distance that enters in the multipole expansion of the soft-photon emission in the o-Ps $\rightarrow 3 \gamma$ decay amplitude is determined by the falloff of the intermediate Green function rather than by the size of the Ps atom, so the series of multipoles is actually an expansion in powers of $\omega r \sim \sqrt{\omega / m}$ which can be used as long as $\omega \ll m$. Note also that for the intermediate pair, an iterative computation of the Coulomb Green function, $G=G^{f}+G^{f} V_{C} G^{f}+\ldots$, with $G^{f}$ the free Green function, shows that adding a Coulomb exchange generates a term

$$
\int d^{3} \mathbf{x} V_{C} G^{f} \sim \frac{\alpha m}{\kappa} \sim \alpha \sqrt{\frac{m}{\omega}} \ll 1, \quad \text { for } \quad m \alpha^{2} \ll \omega \ll m,
$$

as $G^{f} \sim m \exp (-\kappa r) / r$. Therefore the Coulomb interaction can be treated perturbatively in the virtual $e^{+} e^{-}$system after soft photon radiation. The soft-energy region $m \alpha^{2} \ll \omega \ll m$ thus separates the regime where binding effects become essential, from the hard-energy region, where the details of the bound state dynamics are irrelevant.

The NRQED result for the photon energy spectrum in this region, up to NLO reads [2, 3]:

$$
\frac{\mathrm{d} \Gamma_{\mathrm{oPs}}}{\mathrm{d} x}=\frac{2 m \alpha^{6}}{27 \pi}\left[5 x-\frac{14}{3} \alpha \sqrt{x}+\mathscr{O}(\alpha x)\right]
$$

with $x=\omega / m$. The LO term of the NRQED spectrum matches the $\omega \rightarrow 0$ limit of the tree-level QED calculation first done by Ore and Powell [4]. The $\alpha \sqrt{x}$ correction also agrees with the leading term in the $\omega \rightarrow 0$ limit of the 1-loop QED result, as shown in Ref. [2] by expanding the analytic expression of the exact 1-loop phase-space distribution given by Adkins [5]. 


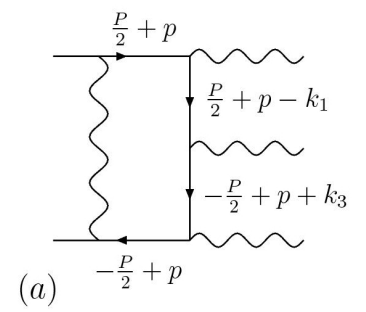

(a)

Figure 2: Ladder and double-vertex graphs contributing to the o-Ps decay amplitude to order $\alpha$.

\section{Threshold expansion of the o-Ps $\rightarrow 3 \gamma$ 1-loop diagrams}

The NLO term in the NRQED result above arises from distances in the $e^{+} e^{-}$system of order $r \sim 1 / \sqrt{m \omega}$, that are much smaller than the size of the Ps atom $r \sim 1 / m \alpha$. Translated into momentum space, this implies that there is a kinematic region where the relative 3-momentum of the non-relativistic $e^{+} e^{-}$pair obeys the non-relativistic relation $p^{0} \sim \mathbf{p}^{2} / m \sim \omega$. This scaling does not correspond to any of the known modes that have been identified in the common applications of non-relativistic EFT's, and should be compared to the scaling of the familiar potential modes, $p^{0} \sim \mathbf{p}^{2} / m \sim m \alpha^{2}$, characteristic of the heavy particles that form the bound state, and that of the soft modes, $p^{0} \sim|\mathbf{p}| \sim m \alpha \gg \mathbf{p}^{2} / m$. The new loop-momentum region has to be taken into account for a successful application of the threshold expansion method [6] to QED and QCD loop diagrams involving a particle-antiparticle system decaying through the emission of a soft photon. In the conventional loop expansion, soft photon radiation from a heavy particle-antiparticle system introduces a soft energy component $\omega \sim m \alpha$ in the zero component of the heavy particle momenta. Non-relativistic poles in massive propagators of the form $\left(p^{0}-\mathbf{p}^{2} / 2 m-\omega\right)^{-1}$ can be thus found in these loops, giving rise to a contribution from the loop-momentum region $p_{0} \sim \mathbf{p}^{2} / m \sim \omega$, which we refer to as soft-radiation region.

To illustrate the latter consider the threshold expansion to the 1-loop o-Ps $\rightarrow 3 \gamma$ amplitude when one of the photons has an energy $\omega_{1} \sim m \alpha$, where the conventional QED perturbative expansion is still valid. For our purposes we can take the electron and positron inside the bound state to be at rest. Then $\omega_{1}$ represents the smallest scale in the diagrams, and the leading term in the $\omega_{1} / \mathrm{m}$ expansion is given by the ladder and double-vertex graphs shown in Fig. 2. The ladder amplitude involves the computation of the 5-point scalar integral:

$$
I_{0}=\int \frac{\left[d^{D} p\right]}{p^{2}\left[(p+P / 2)^{2}-m^{2}\right]\left[(p-P / 2)^{2}-m^{2}\right]\left[\left(p+P / 2-k_{1}\right)^{2}-m^{2}\right]\left[\left(p-P / 2+k_{3}\right)^{2}-m^{2}\right]},
$$

with $P=(2 m, 0)$ the Positronium momentum. When the loop momentum is hard $(p \sim m)$ the corresponding contribution scales as $I_{0}^{(\mathrm{h})} \sim m^{-6}$. However, for $\omega_{1} \rightarrow 0$ the loop-momentum regions where $p \ll m$ give larger contributions. Expanding the propagators for $p \ll m$ we have

$$
I_{0}^{(\mathrm{small})}=-\frac{1}{2 m^{2}} \int \frac{\left[d^{D} p\right]}{\left(p_{0}^{2}-\mathbf{p}^{2}\right)\left(2 m p_{0}-\mathbf{p}^{2}\right)\left(-2 m p_{0}-\mathbf{p}^{2}\right)\left(2 m p_{0}-2 m \omega_{1}-\mathbf{p}^{2}\right)},
$$

which receives contributions from the poles at $p_{0}=\left(-\mathbf{p}^{2} / 2 m+i \varepsilon\right)$ and $p_{0}=(-|\mathbf{p}|+i \varepsilon)$ when we close the integration contour in the upper complex $p_{0}$-plane. From the residue of the former pole 


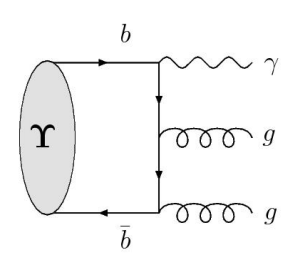

(a)

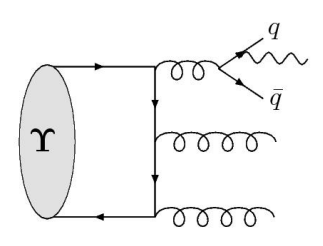

(b)

Figure 3: Leading-order Feynman diagrams for radiative $\Upsilon$ decay.

we obtain the contribution from the soft-radiation region where $p_{0} \sim \mathbf{p}^{2} / m \sim m \omega_{1}$ :

$$
I_{0}^{(\mathrm{s}-\mathrm{r})}=-\frac{i}{16 m^{3}} \int\left[d^{n} \mathbf{p}\right] \frac{1}{\left(\mathbf{p}^{2}\right)^{2}\left(\mathbf{p}^{2}+m \omega_{1}\right)}=\frac{1}{64 \pi m^{3}}\left(m \omega_{1}\right)^{-\frac{3}{2}}
$$

which gives the leading term in the $\omega_{1} / m$ expansion of $I_{0}$. The pole at $p_{0}=(-|\mathbf{p}|+i \varepsilon)$ gives an integral dominated by $\mathbf{p} \sim m \omega_{1}$ which scales as $\left[d^{D} p\right] /\left(m p_{0}\right)^{5} \sim m^{-5} \omega_{1}^{-1}$, i.e. larger than the hard region one, although suppressed by $\sqrt{\omega_{1} / m}$ with respect the term from the soft-radiation region. It is straightforward to verify that only the 1-loop graphs in Fig. 2 receive contributions from the soft-radiation region (see Ref. [2] for a more detailed discussion). Once propagator numerators and vertex factors are included, the soft-radiation piece in the threshold expansion of the 1-loop $e^{+} e^{-} \rightarrow 3 \gamma$ amplitude reproduces the NLO term in the photon spectrum shown in Eq. (1.2).

\section{The direct photon spectrum in the radiative $\Upsilon$ decay for $\omega \sim m_{b} \alpha_{s}$}

The photon energy spectrum in heavy quarkonium radiative decays receives contributions from two different mechanisms. The direct photon spectrum entails those contributions where the observed photon is radiated off a heavy quark, Fig. 3(a), while the fragmentation contributions are those in which one of the partons fragments and transfers a fraction of its momentum to the photon, Fig. 3(b). The NRQCD factorization approach [7] allows to write down an operator product expansion for the direct photon spectrum in, for example, $\Upsilon$ decays:

$$
\frac{\mathrm{d} \Gamma_{\mathrm{dir}}}{\mathrm{d} x}=\sum_{n} C_{n}(x)\left\langle\Upsilon\left|\mathscr{O}_{n}\right| \Upsilon\right\rangle
$$

where the sum above extends over all $Q \bar{Q}[n]$ configurations that can be found inside the quarkonium, and the $C_{n}(x)$ are short distance Wilson coefficients that can be determined from the annihilation cross section of the on-shell $Q \bar{Q}[n]$ pair as a perturbative series in $\alpha_{s}\left(m_{b}\right)$. The $\mathscr{O}_{n}$ in the long-distance matrix element are NRQCD operators which are organized in powers of the relative velocity of the heavy quarks. At leading order only the color-singlet operator $\mathscr{O}_{1}\left({ }^{3} S_{1}\right)$, that creates and annihilates a quark-antiquark pair in a color-singlet ${ }^{3} S_{1}$ configuration, contributes. The nonperturbative NRQCD matrix element is related to the $\Upsilon$ wave function at the origin

$$
\left\langle\Upsilon\left|\mathscr{O}_{1}\left({ }^{3} S_{1}\right)\right| \Upsilon\right\rangle=\left\langle\Upsilon\left|\phi_{\mathbf{p}}^{\dagger} \sigma^{i} \chi_{-\mathbf{p}} \chi_{-\mathbf{p}^{\prime}}^{\dagger} \sigma^{i} \phi_{\mathbf{p}^{\prime}}\right| \Upsilon\right\rangle=2 N_{c}|\psi(0)|^{2}
$$

The matching coefficient at leading order in $\alpha_{s}$ comes from the tree-level $Q \bar{Q} \rightarrow g g \gamma$ annihilation $[8]\left(x=\omega / m_{b}\right.$ in this case),

$$
C_{1}^{\mathrm{LO}}(x)=\frac{32}{27} \frac{\alpha \alpha_{s}^{2} Q_{b}^{2}}{m_{b}^{2}}\left[\frac{2-x}{x}+\frac{(1-x) x}{(2-x)^{2}}-\frac{2(1-x)^{2} \log (1-x)}{(2-x)^{3}}+\frac{2(1-x) \log (1-x)}{x^{2}}\right] \text {, }
$$




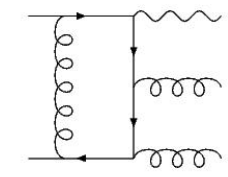

(a)

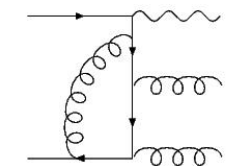

(b)

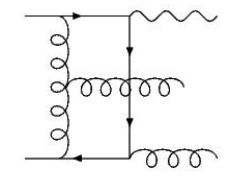

(c)

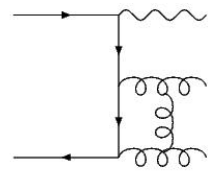

(d)

Figure 4: A sample of the next-to-leading-order diagrams for the direct contributions to radiative $\Upsilon$ decay.

which has identical $x$-dependence to that of the Ore-Powell spectrum for o-Ps [4]. Indeed the factorization approach applies trivially to QED bound states, where everything is calculable perturbatively and the bound state wavefunction reduces to the Coulomb one.

The threshold expansion technique used above for the o-Ps $\rightarrow 3 \gamma$ decay amplitude, can be applied now to calculate the $\mathscr{O}\left(\alpha_{s}\right)$ correction to the short-distance coefficient $C_{1}(x)$ in the region where $\omega \sim m_{b} \alpha_{s}$. A reduced set of the 1-loop diagrams that have to be considered for the virtual corrections $(b \bar{b} \rightarrow \gamma g g)$ is shown in Fig. 3. The abelian topologies are obviously the same as those encountered in the $e^{+} e^{-} \rightarrow 3 \gamma$ 1-loop graphs. Therefore we know that the leading contribution in the $\omega / m_{b}$ expansion comes from the soft-radiation region in the ladder and double-vertex topologies, Figs. 3(a) and 3(b). A quick examination of the pole structure from the propagators in non-abelian diagrams reveals that they do not receive contributions from the soft-radiation region, and thus give subleading terms in $\omega / m_{b}$. Likewise, real corrections corresponding to tree-level diagrams with one more parton in the final state, $b \bar{b} \rightarrow \gamma g g g, \gamma g q \bar{q}$ are not enhanced for $\omega \rightarrow 0$. Therefore we can extract the leading $\mathscr{O}\left(\alpha_{s}\right)$ correction to $C_{1}(x)$ when $\omega \sim m_{b} \alpha_{s}$ from the QED result (1.2) with trivial modifications:

$$
C_{1}^{\mathrm{NLO}}(x)=\left(\frac{8}{27}\right)^{2} \frac{3 \alpha \alpha_{s}^{2} Q_{b}^{2}}{m_{b}^{2}}\left[-\frac{14}{3} \alpha_{s} \sqrt{x}+\mathscr{O}\left(\alpha_{s} x\right)\right] .
$$

From the result (3.3) we obtain a simple expression for the NLO correction to the direct spectrum in the soft-energy region for the radiative $\Upsilon$ decay:

$$
\frac{1}{\Gamma_{\mathrm{dir}}^{\mathrm{LO}}} \frac{\mathrm{d} \Gamma_{\mathrm{dir}}^{\mathrm{NLO}}}{\mathrm{d} x}=-\frac{56}{27} \frac{1}{\left(\pi^{2}-9\right)} \alpha_{s} \sqrt{x}=\left(\frac{4}{3}\right) \frac{1}{\Gamma_{\mathrm{oPs}}^{\mathrm{LO}}} \frac{\mathrm{d} \Gamma_{\mathrm{oPs}}^{\mathrm{NLO}}}{\mathrm{d} x} .
$$

The factor $4 / 3$ above is just given by the ratio between the colour factors in the ladder and tree-level diagrams. The full perturbative $\mathscr{O}\left(\alpha_{s}\right)$ corrections to the $\Upsilon$ photon energy spectrum were calculated numerically in Ref. [9]. We have superimposed the NLO result for the spectrum including the correction (3.4) to the complete $\mathscr{O}\left(\alpha_{s}\right)$ result from Ref. [9] in Fig. 3, where $\alpha_{s}=0.2$. As expected, our result can only improve the LO prediction for $x \lesssim 0.3$, as it was obtained assuming the scaling $x \sim \alpha_{s}$. The agreement with the exact result for small $x$ is remarkably good considering that our formula neglects terms of order $\sqrt{x}$. The approximated NLO direct spectrum becomes negative for $x \lesssim 0.05$. However, at those energies $\left(x \lesssim \alpha_{s}^{2}\right)$ the emission of the photon can produce transitions to virtual bound states and does not longer belong to the short-distance part of the decay [1]. Moreover, we should be aware that the fragmentation contributions to the photon spectrum become important in the low- $x$ region $[10,11]$ (namely for $x \lesssim 0.3$ in $\Upsilon$ decays), and have to be properly taken into account for a computation of the full spectrum. The standard NRQCD factorization also breaks down at large values of the photon energy [12], where one needs to consider also collinear degrees of freedom [13]. 


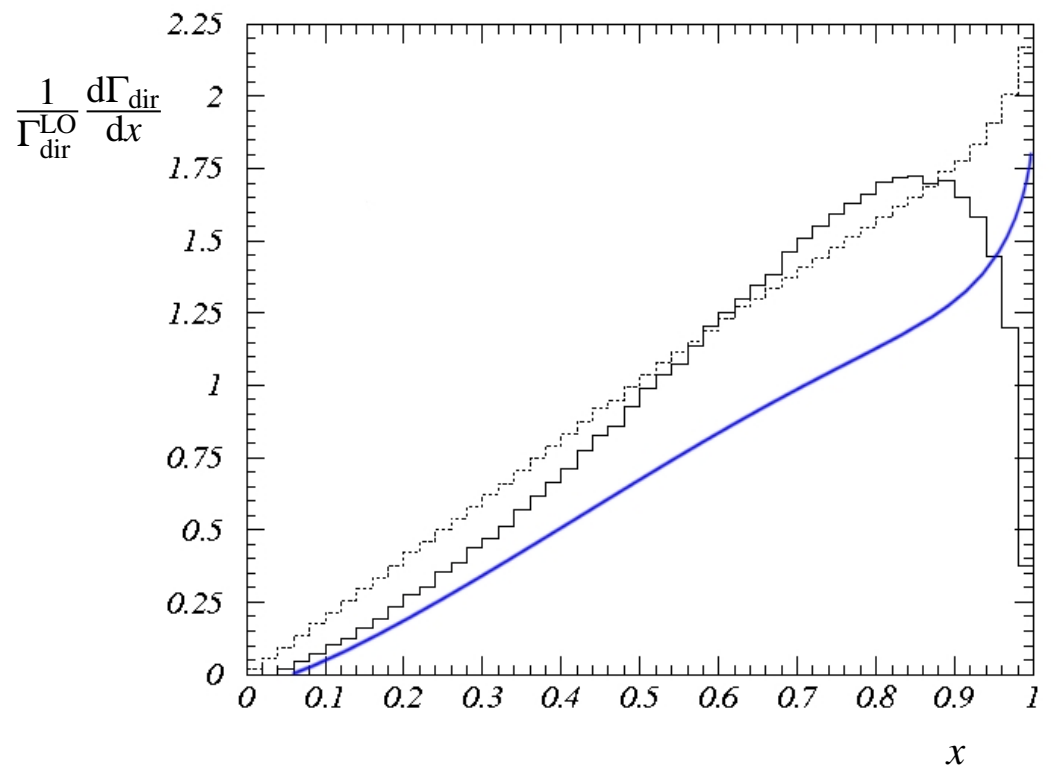

Figure 5: The direct photon energy spectrum in $\Upsilon$ radiative decay. The stepped dotted line is the leadingorder direct photon spectrum, and the solid stepped line includes the full $\mathscr{O}\left(\alpha_{s}\right)$ corrections from Ref. [9]. The solid blue line is the approximated result given by the leading term in the $\omega / m_{b}$ expansion of the $\mathscr{O}\left(\alpha_{s}\right)$ corrections.

\section{References}

[1] A. V. Manohar and P. Ruiz-Femenia, Phys. Rev. D 69 (2004) 053003 [arXiv:hep-ph/0311002].

[2] P. D. Ruiz-Femenia, Nucl. Phys. B 788 (2008) 21 [arXiv:0705.1330 [hep-ph]].

[3] M. B. Voloshin, Mod. Phys. Lett. A 19 (2004) 181 [arXiv:hep-ph/0311204].

[4] A. Ore and J.L. Powell, Phys. Rev. 75 (1949) 1696.

[5] G. S. Adkins, Phys. Rev. A 72, 032501 (2005) [arXiv:hep-ph/0506213].

[6] M. Beneke and V.A. Smirnov, Nucl. Phys. B522, 321 (1998) [arXiv:hep-ph/9711391].

[7] G. T. Bodwin, E. Braaten and G. P. Lepage, Phys. Rev. D 51, 1125 (1995) [Erratum-ibid. D 55, 5853 (1997)] [arXiv:hep-ph/9407339].

[8] K. Koller and T. Walsh, Nucl. Phys. B 140 (1978) 449; S. J. Brodsky, D. G. Coyne, T. A. DeGrand and R. R. Horgan, Phys. Lett. B 73 (1978) 203.

[9] M. Kramer, Phys. Rev. D 60 (1999) 111503 [arXiv:hep-ph/9904416].

[10] S. Catani and F. Hautmann, Nucl. Phys. Proc. Suppl. 39BC (1995) 359 [arXiv:hep-ph/9410394].

[11] F. Maltoni and A. Petrelli, Phys. Rev. D 59 (1999) 074006 [arXiv:hep-ph/9806455].

[12] I. Z. Rothstein and M. B. Wise, Phys. Lett. B 402 (1997) 346 [arXiv:hep-ph/9701404].

[13] S. Fleming and A. K. Leibovich, Phys. Rev. D 67 (2003) 074035 [arXiv:hep-ph/0212094]. X. Garcia i Tormo and J. Soto, Phys. Rev. D 69 (2004) 114006 [arXiv:hep-ph/0401233]. 